\title{
Precise Large Deviations for Random Sums of END Random Variables with Dominated Variation
}

\author{
Yu Chen and Zhihui Qu \\ Department of Statistics and Finance, School of Management, University of Science and Technology of China, Hefei 230026, China
}

Correspondence should be addressed to Yu Chen; cyu@ustc.edu.cn

Received 26 February 2013; Accepted 8 May 2013

Academic Editors: E. Di Nardo and D. Luchinsky

Copyright (C) 2013 Y. Chen and Z. Qu. This is an open access article distributed under the Creative Commons Attribution License, which permits unrestricted use, distribution, and reproduction in any medium, provided the original work is properly cited.

\begin{abstract}
We investigate the precise large deviations for random sums of extended negatively dependent random variables with long and dominatedly varying tails. We find out that the asymptotic behavior of precise large deviations of random sums is insensitive to the extended negative dependence. We apply the results to a generalized dependent compound renewal risk model including premium process and claim process and obtain the asymptotic behavior of the tail probabilities of the claim surplus process.
\end{abstract}

\section{Introduction}

The study of large deviations plays an important role in insurance and finance theory. The aim of this paper is to study precise large deviation probabilities for sequences of dependent and heavy-tailed random variables. Let $\left\{X_{k}, k \geq 1\right\}$ be a sequence of real-valued heavy-tailed random variables with common distribution function $F$ and finite mean $\mu$. We denote its tail by $\bar{F}=1-F$. We say that $X$ (or its distribution $F$ ) is heavy-tailed if it has no exponential moments. Suppose that $\{N(t), t \geq 0\}$ is a nonnegative integer-valued process independent of the sequence $\left\{X_{k}, k \geq 1\right\}$ with mean function $\lambda(t)=E N(t)$ which tends to $\infty$ as $t \rightarrow \infty$. For random sums of the type

$$
S_{N(t)}=\sum_{k=1}^{N(t)} X_{k}, \quad t \geq 0, \quad\left(\sum_{k=1}^{0} X_{k}:=0\right),
$$

we aim to study the precise large deviation probabilities

$$
P\left(S_{N(t)}-E S_{N(t)}>x\right)=P\left(S_{N(t)}-\mu \lambda(t)>x\right) .
$$

Some earlier work, for the case $\left\{X_{k}, k \geq 1\right\}$ are independent, we refer the reader to see Cline and Hsing [1], Klüppelberg and Mikosch [2], Tang et al. [3], Ng et al. [4], Konstantinides and Loukissas [5] and Loukissas [6], and so forth; for the case $\left\{X_{k}, k \geq 1\right\}$ are negatively dependent, see Chen and Zhang [7], Tang [8], Konstantinides and Loukissas [5], Chen et al. [9] and Wang et al. [10], and so forth.
One of the main concepts we use is the extended negative dependence, which was first introduced by Liu [11] and Chen et al. [9]. The definition of this dependence structure is given in the following.

Definition 1. We call random variables $\left\{X_{k}, k \geq 1\right\}$ extended negatively dependent (END), if there exists a constant $M>0$ such that

$$
\begin{aligned}
& P\left(X_{1} \leq x_{1}, \ldots, X_{n} \leq x_{n}\right) \leq M \prod_{k=1}^{n} P\left(X_{k} \leq x_{k}\right), \\
& P\left(X_{1}>x_{1}, \ldots, X_{n}>x_{n}\right) \leq M \prod_{k=1}^{n} P\left(X_{k}>x_{k}\right)
\end{aligned}
$$

hold for each $n \geq 1$ and all $x_{1}, \ldots, x_{n}$.

Recall that if $M=1$ for any integer $n \geq 1$ in (3) and (4), then random variables $\left\{X_{k}, k \geq 1\right\}$ are called lower negatively dependent (LND) and upper negatively dependent (UND), respectively. $\left\{X_{k}, k \geq 1\right\}$ are called negatively dependent (ND) if both (3) and (4) hold, so an ND sequence must be an END sequence. From Liu [11], the END structure can reflect a negative dependence structure but also a positive one to some extent, so the END structure is substantially more comprehensive than the ND structure. 
In presence of END structure, Liu [11] obtained that the precise large deviations of partial sums with consistent variation are insensitive to the dependence structure. Chen et al. [9] extended the previous results of Liu [11] to random sums with consistently varying tails. Wang et al. [10] considered a wider dependent structure and investigated the precise large deviations for the partial sums with dominatedly varying tails.

The basic assumption of this paper is that $\left\{X_{k}, k \geq 1\right\}$ is a sequence of real-valued END random variables with common heavy-tailed distribution $F$ and finite mean $\mu$. We consider a wider heavy-tailed distribution class than consistently varying class, the intersection of long-tailed class, and dominatedly varying-tailed class. The main aim of this paper is to extend the study to certain END cases and find out whether the asymptotic behavior of precise large deviations is insensitive to the extended negative dependence.

The rest of the paper is organized as follows. After simply reviewing some subclasses of heavy-tailed distributions and giving some lemmas needed to prove the theorem in Section 2, we derive our main result of precise large deviations for random sums in Section 3 and apply the result to the dependent compound renewal risk model in Section 4.

\section{Preliminaries}

2.1. Heavy-Tailed Distribution Classes. To formulate our main results we need to introduce some notations and assumptions. Throughout this paper we restrict ourselves to the case that $\left\{X_{k}, k \geq 1\right\}$ is a sequence of heavy-tailed random variables. In risk theory, heavy-tailed distributions are often used to model large claim amounts. They play a key role in some fields such as insurance, financial mathematics, and queueing theory. Next, we recall some important subclasses of heavy-tailed distributions. $\mathscr{D}$, if

A distribution function (d.f.) $F$ is said to belong to class

$$
\limsup _{x \rightarrow \infty} \frac{\bar{F}(x y)}{\bar{F}(x)}<\infty
$$

holds for some $0<y<1$; denote that $F \in \mathscr{D}$. Such a d.f. $F$ is said to have a dominatedly varying tail.

A d.f. $F$ is said to belong to class $\mathscr{L}$, if

$$
\lim _{x \rightarrow \infty} \frac{\bar{F}(x+y)}{\bar{F}(x)}=1
$$

holds for all $y \in(-\infty,+\infty)$; denote that $F \in \mathscr{L}$. Such a d.f. $F$ is said to have a long tail.

A d.f. $F$ is said to belong to class $\mathscr{C}$, if

$$
\lim _{y \searrow 1} \liminf _{x \rightarrow \infty} \frac{\bar{F}(x y)}{\bar{F}(x)}=1,
$$

or equivalently, $\quad \lim _{y>1} \limsup _{x \rightarrow \infty} \frac{\bar{F}(x y)}{\bar{F}(x)}=1$.

Denote that $F \in \mathscr{C}$. Now the d.f. $F$ is said to have a consistently varying tail.
It is well known that the previous heavy-tailed distribution classes have the following relationship:

$$
\mathscr{C} \subset \mathscr{L} \cap \mathscr{D} \subset \mathscr{L}
$$

For more details about heavy-tailed class in the context of insurance and finance, we refer the reader to Embrechts et al. [12], Rolski et al. [13], and Tang and Tsitsiashvili [14], among others.

For a d.f. F, we define

$$
\begin{gathered}
\mathbb{J}_{F}=\inf \left\{-\frac{\log \bar{F}_{*}(y)}{\log y}, y>1\right\} \\
\text { with } \bar{F}_{*}(y)=\liminf _{x \rightarrow \infty} \frac{\bar{F}(x y)}{\bar{F}(x)} \quad \text { for } y>1,
\end{gathered}
$$

and $L_{F}=\lim _{y \downarrow 1} \bar{F}_{*}(y)$; we call $\rrbracket_{F}$ the (upper) Matuszewska index of the d.f. F. From Bingham et al. [15, Chapter 2.1], we know that the following assertions are equivalent:
(i) $F \in \mathscr{D}$;
(ii) $0<L_{F} \leq 1$;
(iii) $\rrbracket_{F}<\infty$.

From the definition of the class $\mathscr{C}$, it holds that $F \in \mathscr{C}$ if and only if $L_{F}=1$. Denote that

$$
\gamma_{F}(y)=\liminf _{x \rightarrow \infty} \frac{\bar{F}(x+y)}{\bar{F}(x)}, \quad y \geq 0 .
$$

If $F \in \mathscr{L}$, we know that $\gamma_{F}(y)=1$.

We close this section by explaining some symbols which will be used later. We will use $\lesssim, z, \sim$, and $\asymp$ to connect two positive functions, say $f_{1}(x)$ and $f_{2}(x)$, as follows:

$$
\begin{aligned}
& f_{1}(x)=f_{2}(x) \quad \text { if } 0<\liminf _{x \rightarrow \infty} \frac{f_{1}(x)}{f_{2}(x)} \\
& \leq \limsup _{x \rightarrow \infty} \frac{f_{1}(x)}{f_{2}(x)}<\infty \\
& f_{1}(x) \lesssim f_{2}(x) \quad \text { if } \limsup _{x \rightarrow \infty} \frac{f_{1}(x)}{f_{2}(x)} \leq 1 \text {; } \\
& f_{1}(x) \gtrsim f_{2}(x) \quad \text { if } \liminf _{x \rightarrow \infty} \frac{f_{1}(x)}{f_{2}(x)} \geq 1 \text {; } \\
& f_{1}(x) \sim f_{2}(x) \quad \text { if } \lim _{x \rightarrow \infty} \frac{f_{1}(x)}{f_{2}(x)}=1 \text {. }
\end{aligned}
$$

We say that $f_{1}(x)$ and $f_{2}(x)$ are weakly equivalent if $f_{1}(x) \asymp$ $f_{2}(x)$. Throughout, every limit relation without explicit limit is with respect to $t \rightarrow \infty$.

2.2. Some Lemmas. We will need some lemmas used in the proofs of our theorems. The following lemma is given by Tang [8]. 
Lemma 2. If $F \in \mathscr{D}$, then

(1) for each $p>\rrbracket_{F}$, there exist positive constants $C$ and $D$ such that the inequality

$$
\frac{\bar{F}(x)}{\bar{F}(y)} \leq C\left(\frac{x}{y}\right)^{p}
$$

holds for all $x \geq y \geq D$;

(2) it holds for each $p>\rrbracket_{F}$ that $x^{-p}=o(\bar{F}(x))$.

Lemma 3. If $F \in \mathscr{D}$, then for any fixed $\theta>0, a(t)$ satisfying $a(t) \rightarrow \infty$ as $t \rightarrow \infty$,

$$
L_{F} \bar{F}(x) \lesssim \bar{F}(x+o(a(t))) \lesssim L_{F}^{-1} \bar{F}(x)
$$

holds uniformly for $x \geq \theta a(t)$.

Proof. The proof is analogous to that of Lemma 2.2 of Chen and Zhang [7] with some minor modifications and is omitted.

By Definition 1, the following properties of END sequences can be obtained directly.

Lemma 4. Let $\left\{X_{k}, k \geq 1\right\}$ be a sequence of END random variables, and then

(1) $\left\{f_{k}\left(X_{k}\right), k \geq 1\right\}$ are still END, where $\left\{f_{k}(\cdot), k \geq 1\right\}$ are either all monotone increasing or all monotone decreasing;

(2) for any $n=1,2, \ldots$, there exists a constant $M>0$ such that

$$
E\left(\prod_{k=1}^{n} X_{k}^{+}\right) \leq M \prod_{k=1}^{n} E X_{k}^{+}
$$
sums.

Wang et al. [10] proved the following result for partial

Lemma 5. Let $\left\{X_{k}, k \geq 1\right\}$ be a sequence of END random variables with common distribution $F \in \mathscr{D}$ and finite mean $\mu=0$, satisfying

$$
\begin{gathered}
F(-x)=o(\bar{F}(x)) \quad \text { as } x \longrightarrow \infty, \\
E\left|X_{1}\right|^{r} I_{\left\{X_{1} \leq 0\right\}}<\infty \quad \text { for some } r>1 .
\end{gathered}
$$

Then, for any $\gamma>0$,

$$
L_{F} \leq \liminf _{n \rightarrow \infty} \inf _{x \geq \gamma n} \frac{P\left(S_{n}>x\right)}{n \bar{F}(x)} \leq \limsup _{n \rightarrow \infty} \sup _{x \geq \gamma n} \frac{P\left(S_{n}>x\right)}{n \bar{F}(x)} \leq L_{F}^{-1} .
$$

Lemma 6. Let $\left\{X_{k}, k \geq 1\right\}$ be a sequence of END random variables with common distribution $F \in \mathscr{D}$ and finite mean $\mu$, satisfying

$$
\begin{gathered}
F(-x)=o(\bar{F}(x)) \quad \text { as } x \longrightarrow \infty, \\
E\left|X_{1}\right|^{r} I_{\left\{X_{1} \leq 0\right\}}<\infty \quad \text { for some } r>1 .
\end{gathered}
$$

Then, for any $\gamma>0$,

$$
\begin{aligned}
L_{F} \gamma_{F}(|\mu|) & \leq \liminf _{n \rightarrow \infty} \inf _{x \geq \gamma n} \frac{P\left(S_{n}-n \mu>x\right)}{n \bar{F}(x)} \\
& \leq \limsup _{n \rightarrow \infty} \sup _{x \geq \gamma n} \frac{P\left(S_{n}-n \mu>x\right)}{n \bar{F}(x)} \leq L_{F}^{-1} \gamma_{F}^{-2}(-\mu \wedge 0) .
\end{aligned}
$$

Proof. Let $X_{k}^{\prime}=X_{k}-\mu, k \geq 1$, and denote its distribution by $F^{\prime}$. It is easy to see that $F^{\prime}(x)=F(x+\mu)$ and

$$
\nabla_{F}(y)=\liminf _{x \rightarrow \infty} \frac{\bar{F}(x+y)}{\bar{F}(x)}=\liminf _{x \rightarrow \infty} \frac{\bar{F}(x+\mu+y)}{\bar{F}(x+\mu)}=\gamma_{F^{\prime}}(y) .
$$

(i) Consider $\mu>0$.

By $F \in \mathscr{D}$ and $F^{\prime}(x)=F(x+\mu)$, it is easy to see that $F^{\prime}(x) \in \mathscr{D}$. In fact for any $0<y<1$,

$$
\begin{aligned}
\limsup _{x \rightarrow \infty} \frac{\bar{F}^{\prime}(x y)}{\bar{F}^{\prime}(x)} & =\limsup _{x \rightarrow \infty} \frac{\bar{F}(x y+\mu)}{\bar{F}(x+\mu)} \\
& \leq \limsup _{x \rightarrow \infty} \frac{\bar{F}((x+\mu) y)}{\bar{F}(x+\mu)}<\infty .
\end{aligned}
$$

By the definition $\gamma_{F}(y)$, we see that

$$
\begin{aligned}
L_{F^{\prime}}(y) & =\lim _{y \downarrow l} \liminf _{x \rightarrow \infty} \frac{\bar{F}(x y+\mu)}{\bar{F}(x+\mu)} \\
& \geq \lim _{y \downarrow} \liminf _{x \rightarrow \infty} \frac{\bar{F}((x+\mu) y)}{\bar{F}(x+\mu)}=L_{F}(y) .
\end{aligned}
$$

Thus, by Lemma 5, we have

$$
\begin{aligned}
& \limsup _{n \rightarrow \infty} \sup _{x \geq \gamma n} \frac{P\left(S_{n}-n \mu>x\right)}{n \bar{F}(x)} \limsup _{n \rightarrow \infty} \sup _{x \geq \gamma n} \frac{P\left(S_{n}-n \mu>x\right)}{n \bar{F}(x+\mu)} \frac{\bar{F}(x+\mu)}{\bar{F}(x)} \\
& \quad \leq \limsup _{n \rightarrow \infty} \sup _{x \geq \gamma n} \frac{P\left(S_{n}-n \mu>x\right)}{n \bar{F}(x+\mu)} \leq L_{F^{\prime}}^{-1} \leq L_{F}^{-1}, \\
& \liminf _{n \rightarrow \infty} \inf _{x \geq \gamma n} \frac{P\left(S_{n}-n \mu>x\right)}{n \bar{F}(x)} \\
&=\liminf _{n \rightarrow \infty} \inf _{x \geq \gamma n} \frac{P\left(S_{n}-n \mu>x\right)}{n \bar{F}(x+\mu)} \frac{\bar{F}(x+\mu)}{\bar{F}(x)} \\
& \geq \liminf _{n \rightarrow \infty} \inf _{x \geq \gamma n} \frac{P\left(S_{n}-n \mu>x\right)}{n \bar{F}(x+\mu)} \liminf _{x \rightarrow \infty} \frac{\bar{F}(x+\mu)}{\bar{F}(x)} \\
& \geq L_{F^{\prime}} \gamma_{F}(\mu) \geq L L_{F} \gamma_{F}(\mu) .
\end{aligned}
$$


(ii) Consider $\mu<0$.

First we shows that $F^{\prime}(x) \in \mathscr{D}$. For large $x$ there exist $\epsilon$ such that $\mu>-\epsilon x$. For some $y>\epsilon$ and $F \in \mathscr{D}$,

$$
\begin{aligned}
\limsup _{x \rightarrow \infty} \frac{\bar{F}^{\prime}(x y)}{\bar{F}^{\prime}(x)} & =\limsup _{x \rightarrow \infty} \frac{\bar{F}(x y+\mu)}{\bar{F}(x)} \\
& \leq \limsup _{x \rightarrow \infty} \frac{\bar{F}((y-\epsilon) x)}{\bar{F}(x+\mu)}<\infty .
\end{aligned}
$$

By the definition $\gamma_{F}(y)$, we see that

$$
\begin{aligned}
L_{F^{\prime}}(y) & =\lim _{y \downarrow 1} \liminf _{x \rightarrow \infty} \frac{\bar{F}(x y+\mu)}{\bar{F}(x+\mu)} \\
& \geq \lim _{y \downarrow 1} \liminf _{x \rightarrow \infty} \frac{\bar{F}(x y)}{\bar{F}(x)} \liminf _{x \rightarrow \infty} \frac{\bar{F}(x)}{\bar{F}(x+\mu)}=L_{F} \gamma_{F}(-\mu) .
\end{aligned}
$$

Similarly, by Lemma 5, we have

$$
\begin{aligned}
& \limsup _{n \rightarrow \infty} \sup _{x \geq \gamma n} \frac{P\left(S_{n}-n \mu>x\right)}{n \bar{F}(x)} \limsup _{n \rightarrow \infty} \sup _{x \geq \gamma n} \frac{P\left(S_{n}-n \mu>x\right)}{n \bar{F}(x+\mu)} \frac{\bar{F}(x+\mu)}{\bar{F}(x)} \\
& \leq \limsup _{n \rightarrow \infty} \sup _{x \geq \gamma n} \frac{P\left(S_{n}-n \mu>x\right)}{n \bar{F}(x+\mu)} \limsup _{n \rightarrow \infty} \frac{\bar{F}(x+\mu)}{\bar{F}(x)} \\
& \leq L_{F^{\prime}}^{-1}\left(\liminf _{x \rightarrow \infty} \frac{\bar{F}(x)}{\bar{F}(x+\mu)}\right)^{-1}=L_{F}^{-1} \gamma_{F}^{-2}(-\mu), \\
& \liminf _{n \rightarrow \infty} \inf _{x \geq \gamma n} \frac{P\left(S_{n}-n \mu>x\right)}{n \bar{F}(x)} \\
&=\liminf _{n \rightarrow \infty} \inf _{x \geq \gamma n} \frac{P\left(S_{n}-n \mu>x\right)}{n \bar{F}(x+\mu)} \frac{\bar{F}(x+\mu)}{\bar{F}(x)} \\
& \quad \geq \liminf _{n \rightarrow \infty} \inf _{x \geq \gamma n} \frac{P\left(S_{n}-n \mu>x\right)}{n \bar{F}(x+\mu)} \liminf _{x \rightarrow \infty} \frac{\bar{F}(x+\mu)}{\bar{F}(x)} \\
& \geq L_{F^{\prime}} \geq L_{F} \gamma_{F}(-\mu) .
\end{aligned}
$$

Lemma 7. Let $\left\{X_{k}, k \geq 1\right\}$ be END with common distribution $F \in \mathscr{D}$ and mean 0, satisfying (18). Denote that $S_{n}=$ $\sum_{k=1}^{n} X_{k}, n \geq 1$. Then, for each fixed $\gamma>0$ and some $C=C(\gamma)$ irrespective to $x$ and $n$, the inequality

$$
P\left(S_{n}>x\right) \leq \operatorname{Cn} \bar{F}(x)
$$

holds uniformly for all $x \geq \gamma n$ and $n=1,2, \ldots$.

Proof. Firstly, by Lemma 5, we know that

$$
P\left(S_{n}>x\right) \lesssim L_{F}^{-1} n \bar{F}(x)
$$

holds uniformly for all $x \geq \gamma n$. Moreover, for each fixed positive integer $n_{0}$, by Lemma 2 , the inequality

$$
\begin{aligned}
P\left(\sum_{k=1}^{n} X_{k}>x\right) & \leq P\left(\bigcup_{i=1}^{n}\left\{X_{k}>\frac{x}{n}\right\}\right) \leq n \bar{F}\left(\frac{x}{n_{0}}\right) \\
& \leq C\left(n_{0}\right) n \bar{F}(x)
\end{aligned}
$$

holds for some $C\left(n_{0}\right)>0$, all $x \geq 0$, and all $n=1,2, \ldots, n_{0}$. Hence, we complete the proof.

Lemma 8. Let $\left\{X_{k}, k \geq 1\right\}$ be a sequence of END random variables with common distribution $F$ and finite mean $\mu$; if $0<\mu_{+}=E X_{1} I_{\left\{X_{1}>0\right\}}<\infty$, then for any $v>0$ and some $C=C(v)>0$, the inequality

$$
P\left(\sum_{k=1}^{n} X_{k}>x\right) \leq n P\left(X_{1}>\frac{x}{v}\right)+C\left(\frac{n}{x}\right)^{v}
$$

holds for all $n=1,2, \ldots$ and $x>0$.

This lemma plays an important role in the proof of Theorem 9, which is Lemma 2.3 of Chen et al. [9].

\section{Precise Large Deviations for Random Sums}

\subsection{Main Theorem}

Theorem 9. Let $\left\{X_{k}, k \geq 1\right\}$ be a sequence of real-valued END random variables with common distribution $F \in \mathscr{L} \cap \mathscr{D}$ and finite mean $\mu$, satisfying (18). Let $\{N(t), t \geq 0\}$ be a nonnegative integer-valued process independent of $\left\{X_{k}, k \geq 1\right\}$, and assume that $N(t)$ satisfies

$$
\text { Assumption } N_{1}: E N(t)^{p} I(N(t)>(1+\delta) \lambda(t))=O(\lambda(t))
$$

for some $p>\rrbracket_{F}$ and

$$
\text { Assumption } \begin{aligned}
N_{2} & : P(N(t) \leq(1-\delta) \lambda(t)) \\
& =o(\lambda(t) \bar{F}(\lambda(t)))
\end{aligned}
$$

for all $0<\delta<1 . S_{N(t)}$ is defined by (1), and then for any $\gamma>0$,

$$
L_{F}^{2} \lambda(t) \bar{F}(x) \lesssim P\left(S_{N(t)}-\mu \lambda(t)>x\right) \lesssim L_{F}^{-2} \lambda(t) \bar{F}(x)
$$

holds uniformly for $x \geq \gamma \lambda(t)$.

Remark 10. From the following proof, it is easy to see that (33) holds uniformly for $x \geq \gamma \lambda(t)$ and $\gamma>|\mu|$ if $N(t)$ just satisfies Assumption $N_{1}$.

Corollary 11. Under the assumptions of Theorem 9 and if $F \in$ $\mathscr{C}$, then for any $\gamma>0$,

$$
P\left(S_{N(t)}-\mu \lambda(t)>x\right) \sim \lambda(t) \bar{F}(x)
$$

holds uniformly for $x \geq \gamma \lambda(t)$.

From Remark 10, if $\mu>0$, Corollary 11 is just the same as Theorem 3.1 of Chen et al. [9]. The previous results extend Chen et al. [9] and Chen and Zhang [7]. 
3.2. Proof of Theorem 9. In the sequel, $C$ always represents an absolute positive constant, which may vary from different places.

In order to prove Theorem 9, we divide the random sum into three parts as follows:

$$
\begin{aligned}
P( & \left.S_{N(t)}-\mu \lambda(t)>x\right) \\
= & \sum_{n=1}^{\infty} P\left(S_{n}-\mu \lambda(t)>x\right) P(N(t)=n) \\
= & \left(\sum_{n<(1-\delta) \lambda(t)}+\sum_{|n-\lambda(t)| \leq \delta \lambda(t)}+\sum_{n>(1+\delta) \lambda(t)}\right) \\
& \times P\left(S_{n}-\mu \lambda(t)>x\right) P(N(t)=n),
\end{aligned}
$$

where $\delta \in(0,1)$ is an arbitrarily fixed number to be specified later.

By Tang et al. [3], Assumption $N_{1}$ implies that

$$
\frac{N(t)}{\lambda(t)} \stackrel{p}{\longrightarrow} 1
$$

then by the dominated convergence theorem,

$$
\lim _{t \rightarrow \infty} E\left[\frac{N(t)}{\lambda(t)} I(|N(t)-\lambda(t)| \leq \delta \lambda(t))\right]=1
$$

Therefore, for any $0<\delta<1$,

$$
\begin{aligned}
& E[N(t) I(N(t)>(1+\delta) \lambda(t))]=o(\lambda(t)), \\
& E[N(t) I(N(t)<(1-\delta) \lambda(t))]=o(\lambda(t)) .
\end{aligned}
$$

Therefore, Theorem 9 originates from the following three lemmas.

Lemma 12. Assume that Assumptions $N_{1}$ and $N_{2}$ hold; then for any $\gamma>0$,

$$
\begin{aligned}
J_{1} & :=\sum_{n<(1-\delta) \lambda(t)} P\left(S_{n}-\mu \lambda(t)>x\right) P(N(t)=n) \\
& =o(\lambda(t) \bar{F}(x))
\end{aligned}
$$

holds uniformly for $x \geq \gamma \lambda(t)$.

Proof. In order to prove this lemma, we consider the following three cases. (i) $\mu \geq 0$ and $\gamma>0$.

Choose $0<\delta<1$ such that $\delta \mu<\gamma$. Note that $n<(1-$ $\delta) \lambda(t), x \geq \gamma \lambda(t)>(\gamma /(1-\delta)) n$. By Lemma 7 ,

$$
\begin{aligned}
J_{1} & =\sum_{n<(1-\delta) \lambda(t)} P\left(S_{n}-\mu \lambda(t)>x\right) P(N(t)=n) \\
& =\sum_{n<(1-\delta) \lambda(t)} P\left(S_{n}-n \mu>x+\mu(\lambda(t)-n)\right) P(N(t)=n) \\
& \leq \sum_{n<(1-\delta) \lambda(t)} P\left(S_{n}-n \mu>x\right) P(N(t)=n) \\
& \leq C \bar{F}(x) \sum_{n<(1-\delta) \lambda(t)} n P(N(t)=n) \\
& =C \bar{F}(x) E N(t) I(N(t)<(1-\delta) \lambda(t)) \\
& =o(\lambda(t) \bar{F}(x)),
\end{aligned}
$$

and the last equality holds by relation (39).

(ii) $\mu<0$ and $\gamma>|\mu|$.

Choose $\delta$ such that $(\gamma+\mu) /(1-\delta)-\mu>0$. Note that $x+\mu \lambda(t)-n \mu \geq((\gamma+\mu) /(1-\delta)-\mu) n$. Mimicking the proof of (i) and by Lemma 7, we have

$$
\begin{aligned}
J_{1} & \leq \sum_{n<(1-\delta) \lambda(t)} C n \bar{F}(x+\mu \lambda(t)-n \mu) P(N(t)=n) \\
& \leq C \bar{F}(x+\mu \lambda(t)) E N(t) I(N(t)<(1-\delta) \lambda(t)) \\
& =o(\lambda(t) \bar{F}(x)),
\end{aligned}
$$

where in the last step, we used the relation $\bar{F}(x+\mu \lambda(t)) \leq$ $\bar{F}((1+\mu / \gamma) x) \asymp \bar{F}(x)$.

(iii) $\mu<0$ and $\gamma \in(0,|\mu|]$.

Let $\gamma_{1}>|\mu|$. For $x \geq \gamma_{1} \lambda(t)$, we have $x+\mu \lambda(t)-n \mu>|\mu| n$. By Lemma 7,

$$
\begin{aligned}
J_{1} & \leq \sum_{n<(1-\delta) \lambda(t)} C n \bar{F}(x+\mu \lambda(t)-n \mu) P(N(t)=n) \\
& \leq C \bar{F}(x+\mu \lambda(t)) \sum_{n<(1-\delta) \lambda(t)} n P(N(t)=n) \\
& =C \bar{F}(x+\mu \lambda(t)) E N(t) I(N(t)<(1-\delta) \lambda(t)) \\
& =o(\lambda(t) \bar{F}(x)),
\end{aligned}
$$

where the last step can be verified as $\bar{F}(x+\mu \lambda(t)) \leq \bar{F}((1+$ $\left.\left.\mu / \gamma_{1}\right) x\right) \asymp \bar{F}(x)$.

For $\gamma \lambda(t) \leq x<\gamma_{1} \lambda(t)$, note that $\bar{F}(x) \geq \bar{F}\left(\gamma_{1} \lambda(t)\right) \asymp$ $\bar{F}(\lambda(t))$. Hence, by Assumption $N_{2}$, the inequality

$$
\begin{aligned}
J_{1} & \leq P(N(t) \leq(1-\delta) \lambda(t))=o(1) \lambda(t) \bar{F}(\lambda(t)) \\
& =o(\lambda(t) \bar{F}(x))
\end{aligned}
$$


still holds uniformly for all $\gamma \lambda(t) \leq x<\gamma_{1} \lambda(t)$. As a result, the relation

$$
J_{1}=o(\lambda(t) \bar{F}(x))
$$

holds uniformly for all $x \geq \gamma \lambda(t)$.

Lemma 13. Assume that Assumptions $N_{1}$ and $N_{2}$ hold; then for any $\gamma>0$,

$$
\begin{aligned}
L_{F}^{2} \lambda(t) \bar{F}(x) & \leq J_{2} \\
& :=\sum_{|n-\lambda(t)| \leq \delta \lambda(t)} P\left(S_{n}-\mu \lambda(t)>x\right) P(N(t)=n) \\
& \leq L_{F}^{-2} \lambda(t) \bar{F}(x)
\end{aligned}
$$

holds uniformly for $x \geq \gamma \lambda(t)$.

Proof. Similar to the proof of Lemma 12, we split into three cases.

(i) $\mu \geq 0$ and $\gamma>0$.

Firstly, for $|n-\lambda(t)| \leq \delta \lambda(t)$, then $\lambda(t)-n \geq-\delta \lambda(t)$. For $x \geq \gamma \lambda(t)$,

$$
\begin{aligned}
J_{2} & =\sum_{|n-\lambda(t)| \leq \delta \lambda(t)} P\left(S_{n}-\mu \lambda(t)>x\right) P(N(t)=n) \\
& =\sum_{|n-\lambda(t)| \leq \delta \lambda(t)} P\left(S_{n}-n \mu>x+\mu(\lambda(t)-n)\right) P(N(t)=n) \\
& \leq \sum_{|n-\lambda(t)| \leq \delta \lambda(t)} P\left(S_{n}-n \mu>x-\mu \delta \lambda(t)\right) P(N(t)=n) \\
& \leq \sum_{|n-\lambda(t)| \leq \delta \lambda(t)} P\left(S_{n}-n \mu>\left(1-\frac{\mu \delta}{\gamma}\right) x\right) P(N(t)=n) .
\end{aligned}
$$

By Lemma 6, for any $\epsilon>0$ and sufficient large $t, x \geq \gamma \lambda(t) \geq$ $(\gamma /(1+\delta)) n$,

$$
P\left(S_{n}-n \mu>\left(1-\frac{\mu \delta}{\gamma}\right) x\right) \leq(1+\epsilon) L_{F}^{-1} n \bar{F}\left(\left(1-\frac{\mu \delta}{\gamma}\right) x\right) .
$$

Then,

$$
\begin{aligned}
J_{2} \leq & (1+\epsilon) L_{F}^{-1} \bar{F}\left(\left(1-\frac{\mu \delta}{\gamma}\right) x\right) \sum_{|n-\lambda(t)| \leq \delta \lambda(t)} n P(N(t)=n) \\
= & (1+\epsilon) L_{F}^{-1} \bar{F}\left(\left(1-\frac{\mu \delta}{\gamma}\right) x\right) E N(t) I \\
& \times(|N(t)-\lambda(t)| \leq \delta \lambda(t)) ;
\end{aligned}
$$

thus by $F \in \mathscr{L} \cap \mathscr{D}$, letting $\delta \rightarrow 0$, we have $\lim _{\delta \rightarrow 0} \bar{F}((1-$ $\mu \delta / \gamma) x) / \bar{F}(x)=L_{F}^{-1}$. Combining this and (37),

$$
\limsup _{t \rightarrow \infty} \sup _{x \geq \gamma \lambda(t)} \frac{J_{2}}{\lambda(t) \bar{F}(x)} \leq L_{F}^{-2} .
$$

On the other hand, by the similar way, for $|n-\lambda(t)| \leq \delta \lambda(t)$, then $\lambda(t)-n \leq \delta \lambda(t)$

$$
\begin{aligned}
J_{2} & =\sum_{|n-\lambda(t)| \leq \delta \lambda(t)} P\left(S_{n}-\mu \lambda(t)>x\right) P(N(t)=n) \\
\geq & \sum_{|n-\lambda(t)| \leq \delta \lambda(t)} P\left(S_{n}-n \mu>x+\mu \delta \lambda(t)\right) P(N(t)=n) \\
\geq & \sum_{|n-\lambda(t)| \leq \delta \lambda(t)} P\left(S_{n}-n \mu>\left(1+\frac{\mu \delta}{\gamma}\right) x\right) P(N(t)=n) \\
\geq & (1-\epsilon) L_{F} \gamma_{F}(\mu) \bar{F}\left(\left(1+\frac{\delta \mu}{\gamma}\right) x\right) E N(t) I \\
& \quad \times(|N(t)-\lambda(t)| \leq \delta \lambda(t)) .
\end{aligned}
$$

We have

$$
\liminf _{t \rightarrow \infty} \inf _{x>\gamma \lambda(t)} \frac{J_{2}}{\lambda(t) \bar{F}(x)} \geq L_{F}^{2} \gamma_{F}(\mu) .
$$

Combining (50) and (52), we complete the proof of the lemma in this case.

(ii) $\mu<0$ and $\gamma>|\mu|$.

Similarly, for any $\epsilon>0$ and sufficient $t, x \geq \gamma \lambda(t)$,

$$
\begin{aligned}
J_{2} \leq & \sum_{|n-\lambda(t)| \leq \delta \lambda(t)}(1+\epsilon) L_{F}^{-1} n \bar{F}(x+\mu \lambda(t)-n \mu) P(N(t)=n) \\
\leq & (1+\epsilon) L_{F}^{-1} \bar{F}(x+\mu \lambda(t)-\mu(1-\delta) \lambda(t)) \\
& \times \sum_{|n-\lambda(t)| \leq \delta \lambda(t)} n P(N(t)=n) \\
\leq & (1+\epsilon) L_{F}^{-1} \gamma_{F}^{-2}(-\mu) \bar{F}\left(\left(1+\frac{\delta \mu}{\gamma}\right) x\right) E N(t) I \\
& \times(|N(t)-\lambda(t)| \leq \delta \lambda(t)),
\end{aligned}
$$$$
\begin{aligned}
J_{2} \geq & (1-\epsilon) L_{F} \gamma_{F}(-\mu) \bar{F}\left(\left(1-\frac{\delta \mu}{\gamma}\right) x\right) E N(t) I \\
& \times(|N(t)-\lambda(t)| \leq \delta \lambda(t)) .
\end{aligned}
$$

Analogously, we can obtain

$$
\begin{aligned}
& \limsup _{t \rightarrow \infty} \sup _{x \geq \gamma \lambda(t)} \frac{J_{2}}{\lambda(t) \bar{F}(x)} \leq L_{F}^{-2} \gamma_{F}^{-2}(-\mu), \\
& \liminf _{t \rightarrow \infty} \inf _{x>\gamma \lambda(t)} \frac{J_{2}}{\lambda(t) \bar{F}(x)} \geq L_{F}^{2} \gamma_{F}(-\mu) .
\end{aligned}
$$


(iii) $\mu<0$ and $\gamma \in(0,|\mu|]$.

Since $\gamma \in(0,|\mu|]$, note that $x+\mu \lambda(t)-n \mu \geq(\gamma+\mu) \lambda(t)-$ $\mu n \geq((\gamma+\mu) /(1-\delta)-\mu) n$, and it follows from Lemma 6 that for all $x \geq \gamma \lambda(t)$,

$$
\begin{aligned}
J_{2} \leq & \sum_{|n-\lambda(t)| \leq \delta \lambda(t)}(1+\epsilon) L_{F}^{-1} n \bar{F}(x+\mu \lambda(t)-n \mu) P(N(t)=n) \\
\leq & (1+\epsilon) L_{F}^{-1} \bar{F}(x+\mu \lambda(t)-\mu(1-\delta) \lambda(t)) \\
& \times \sum_{|n-\lambda(t)| \leq \delta \lambda(t)} n P(N(t)=n) \\
\leq & (1+\epsilon) L_{F}^{-1} \bar{F}\left(\left(1+\frac{\delta \mu}{\gamma}\right) x\right) E N(t) I \\
& \times(|N(t)-\lambda(t)| \leq \delta \lambda(t)) .
\end{aligned}
$$

On the other hand, for $x \geq \gamma \lambda(t) \geq(\gamma /(1+\delta)) n$,

$$
\begin{aligned}
J_{2} \geq & (1-\epsilon) L_{F} \sum_{|n-\lambda(t)| \leq \delta \lambda(t)} n \bar{F}\left(\left(1-\frac{\mu \delta}{\gamma}\right) x\right) P(N(t)=n) \\
= & (1-\epsilon) L_{F} \bar{F}\left(\left(1-\frac{\mu \delta}{\gamma}\right) x\right) E N(t) I \\
& \times(|N(t)-\lambda(t)| \leq \delta \lambda(t)) .
\end{aligned}
$$

Similarly, both (50) and (52) hold in this case.

Lemma 14. Assume that Assumptions $N_{1}$ and $N_{2}$ hold, and then for any $\gamma>0$,

$$
\begin{aligned}
J_{3} & :=\sum_{n>(1+\delta) \lambda(t)} P\left(S_{n}-\mu \lambda(t)>x\right) P(N(t)=n) \\
& =o(\lambda(t) \bar{F}(x))
\end{aligned}
$$

holds uniformly for $x>\gamma \lambda(t)$.

Proof. We prove this lemma by splitting into three cases like the former lemmas' proof.

(i) $\mu \geq 0$ and $\gamma>0$.

For $p>\rrbracket_{F} \geq 1$, by Lemma 8 , there exists a constant $C>0$ such that

$$
\begin{aligned}
J_{3} & =\sum_{n>(1+\delta) \lambda(t)} P\left(S_{n}-\mu \lambda(t)>x\right) P(N(t)=n) \\
& \leq \sum_{n>(1+\delta) \lambda(t)} P\left(S_{n}>x\right) P(N(t)=n) \\
& \leq \sum_{n>(1+\delta) \lambda(t)}\left(n \bar{F}\left(\frac{x}{p}\right)+C\left(\frac{n}{x}\right)^{p}\right) P(N(t)=n) \\
& =\bar{F}\left(\frac{x}{p}\right) E N(t) I(N(t)>(1+\delta) \lambda(t))
\end{aligned}
$$

$$
\begin{aligned}
& +C x^{-p} \operatorname{EN}(t)^{p} I(N(t)>(1+\delta) \lambda(t)) \\
= & \bar{F}(x) E N(t) I(N(t)>(1+\delta) \lambda(t)) \\
& +x^{-p} \operatorname{EN}(t)^{p} I(N(t)>(1+\delta) \lambda(t)) \\
= & o(\lambda(t) \bar{F}(x)),
\end{aligned}
$$

where in the last step we have used Lemma 2, Assumption $N_{1}$ and relation (38).

(ii) $\mu<0$ and $\gamma>|\mu|$.

Similarly, by Lemma 8 , there exists a constant $C>0$ such that

$$
\begin{aligned}
J_{3} \leq & \sum_{n>(1+\delta) \lambda(t)} P\left(S_{n}>\left(1+\frac{\mu}{\gamma}\right) x\right) P(N(t)=n) \\
\leq & \sum_{n>(1+\delta) \lambda(t)}\left(n \bar{F}\left(\frac{(1+\mu / \gamma) x}{p}\right)\right. \\
& \left.+C\left(\frac{n}{(1+\mu / \gamma) x}\right)^{p}\right) P(N(t)=n) \\
= & \bar{F}\left(\frac{(1+\mu / \gamma) x}{p}\right) E N(t) I(N(t)>(1+\delta) \lambda(t)) \\
& +C\left(\left(1+\frac{\mu}{\gamma}\right) x\right)^{-p} E N(t)^{p} I(N(t)>(1+\delta) \lambda(t)) \\
= & \bar{F}(x) E N(t) I(N(t)>(1+\delta) \lambda(t)) \\
& +x^{-p} E N(t)^{p} I(N(t)>(1+\delta) \lambda(t)) \\
= & o(\lambda(t) \bar{F}(x)) .
\end{aligned}
$$

(iii) $\mu<0$ and $\gamma \in(0,|\mu|]$.

Note that $x+\mu \lambda(t)-n \mu \geq(\gamma+\mu) \lambda(t)-\mu n \geq((\gamma+\mu) /(1+$ $\delta)-\mu) n$. Therefore, Lemma 6 implies that

$$
\begin{aligned}
J_{3} \leq & \sum_{n>(1+\epsilon) \lambda(t)}(1+\delta) L_{F}^{-1} n \bar{F}(x+\mu \lambda(t)-n \mu) P(N(t)=n) \\
\leq & (1+\epsilon) L_{F}^{-1} \bar{F}(x+\mu \lambda(t)-\mu(1+\epsilon) \lambda(t)) E N(t) I \\
& \times(N(t)>(1+\epsilon) \lambda(t)) \\
= & o(\lambda(t) \bar{F}(x)) .
\end{aligned}
$$

\section{Applications for Random Sums for a Dependent Compound Renewal Risk Model}

4.1. The Dependent Compound Renewal Risk Model. From a realistic point of view, we further generalize such work to a much more realistic model including premium income process. The premium income process depends not only on 
the number of customers who buy the insurance portfolios but also on the premium size process. The risk model has the following structure.

(1) The individual claim sizes $\left\{X_{k}, k \geq 1\right\}$ are END random variables with common distribution $F$ and finite mean $\mu=E X_{1}$.

(2) The number of claims in the interval $[0, t]$ is denoted by $\{N(t), t \geq 0\}$. Suppose that $\{N(t), t \geq 0\}$ is a nonnegative integer-valued process with mean function $\lambda(t)$ which tends to $\infty$ as $t \rightarrow \infty$ and independent of $\left\{X_{k}, k \geq 1\right\}$.

(3) The number of customers who buy the insurance portfolios within the time interval $(0, t]$ is denoted by $\{M(t), t \geq 0\}$. Assume that $\{M(t), t \geq 0\}$ is a strictly stationary renewal counting process with mean function $\operatorname{EM}(t)=\beta t, \beta>0$.

(4) The premium size process $\left\{Y_{k}, k \geq 1\right\}$ is a sequence of nonnegative UND random variables.

Suppose that $u>0$ is the initial reserve of an insurance company. Thus, the risk reserve process $\{R(t), t \geq 0\}$ is given by

$$
R(t)=u+\sum_{k=1}^{M(t)} Y_{k}-\sum_{k=1}^{N(t)} X_{k}, \quad t \geq 0,
$$

while the claim surplus process $\{S(t), t \geq 0\}$ is

$$
S(t)=\sum_{k=1}^{N(t)} X_{k}-\sum_{k=1}^{M(t)} Y_{k}, \quad t \geq 0 .
$$

In addition, the random sequences $\{M(t), t \geq 0\},\left\{Y_{k}, k \geq\right.$ $1\},\left\{X_{k}, k \geq 1\right\}$, and $\{N(t), t \geq 0\}$ are mutually independent. This model (62) is called generalized dependent compound renewal risk model. For this model (62), the net profit condition is that $\mu \lambda(t)<\beta t E Y_{1}$.

Theorem 15. For the generalized dependent compound renewal risk model (62), let $\left\{X_{k}, k \geq 1\right\}$ be a sequence of END random variables with common distribution $F \in \mathscr{L} \cap \mathscr{D}$ and finite mean $\mu$, satisfying (18), and let $\left\{Y_{k}, k \geq 1\right\}$ be a sequence of UND nonnegative random variables. Assume that $N(t)$ satisfies Assumptions $N_{1}$ and $N_{2}$, and then for any $\gamma>E Y_{1}$,

$$
L_{F}^{3} \lambda(t) \bar{F}(x) \lesssim P(S(t)-E S(t)>x) \lesssim L_{F}^{-3} \lambda(t) \bar{F}(x)
$$

holds uniformly for $x \geq \gamma \lambda(t) \vee \gamma \beta t$.

Applying Theorem 9 to the generalized dependent compound renewal risk model (62), we can obtain the previous theorem by the following lemmas.

By the same argument as in Theorem 15, we can easily obtain the following corollary which extends Hu's result [16].

Corollary 16. Under the conditions of Theorem 15, further assume that $N(t)$ is a Poisson process with intensity $\lambda>0$, and then for any $\gamma>0$ with $\gamma \lambda>\beta E Y_{1}$,

$$
L_{F}^{3} \lambda t \bar{F}(x) \lesssim P(S(t)-E S(t)>x) \lesssim L_{F}^{-3} \lambda t \bar{F}(x)
$$

holds uniformly for $x \geq \gamma \lambda t$.
Remark 17. If we assume that $F \in \mathscr{C}$ in Theorem 15 , we have that

$$
P(S(t)-E S(t)>x) \sim \lambda(t) \bar{F}(x)
$$

holds uniformly for $x \geq \gamma \lambda(t) \vee \gamma \beta t$.

From the previous two theorems, we find out that the asymptotic behavior of precise large deviations of random sums is insensitive to the extended negative dependence.

4.2. Proof of Theorem 15. Observing that $\{M(t), t \geq 0\}$ is a renewal counting process, $E M(t)=\beta t$. According to the strong law of large numbers for UND random variables (see Matuła [17, Theorem 1]), when $t \rightarrow \infty$, we have

$$
\frac{1}{t} \sum_{j=1}^{M(t)} Y_{j}=\frac{M(t)}{t} \cdot \frac{1}{M(t)} \sum_{j=1}^{M(t)} Y_{j} \longrightarrow \beta E Y_{1}
$$

Consequently, there is a positive function $\epsilon(t)$ such that $\epsilon(t) \rightarrow 0$, and

$$
P\left(\left|\sum_{j=1}^{M(t)} Y_{j}-\beta t E Y_{1}\right|>\epsilon(t) \beta t E Y_{1}\right)=o(1) .
$$

For convenience, we denote that $J(x ; t)=J(x)=P\left(\sum_{j=1}^{M(t)}\right.$ $\left.Y_{j} \leq x\right)$. For the model (62), we have

$$
\begin{aligned}
& (S(t)-E S(t)>x) \\
& \quad=P\left(S_{N(t)}-\sum_{j=1}^{M(t)} Y_{j}-\mu \lambda(t)+E M(t) E Y_{1}>x\right) \\
& \quad=\int_{0}^{\infty} P\left(S_{N(t)}-\mu \lambda(t)>x-\beta t E Y_{1}+y\right) d J(y) .
\end{aligned}
$$

In order to prove the theorem, we divide this integral into three parts similarly as follows:

$$
\begin{aligned}
\int_{0}^{\infty}= & \int_{y-\beta t E Y_{1}<-\epsilon(t) \beta t E Y_{1}}+\int_{\left|y-\beta t E Y_{1}\right| \leq \epsilon(t) \beta t E Y_{1}} \\
& +\int_{y-\beta t E Y_{1}>\epsilon(t) \beta t E Y_{1}} .
\end{aligned}
$$

We proceed a series of lemmas to prove Theorem 15.

Lemma 18. For $\epsilon(t)$ in (67), one has for $\gamma>E Y_{1}$ that

$$
\begin{aligned}
I_{1}=\int_{y-\beta t E Y_{1}<-\epsilon(t) \beta t E Y_{1}} P\left(S_{N(t)}-\mu \lambda(t)>x\right. \\
\left.\quad-\beta t E Y_{1}+y\right) d J(y) \\
=o(\lambda(t) \bar{F}(x))
\end{aligned}
$$

holds uniformly for $x \geq \gamma \lambda(t) \vee \gamma \beta t$. 
Proof. For $\gamma>E Y_{1}$, then $1-E Y_{1} / \gamma>0$. For $y \geq 0, x \geq$ $\gamma \lambda(t) \vee \gamma \beta t$, then $t \leq x / \gamma \beta$, and applying Theorem 9, we have

$$
\begin{aligned}
I_{1}=\int_{y-\beta t E Y_{1}<-\epsilon(t) \beta t E Y_{1}} P\left(S_{N(t)}-\mu \lambda(t)>x-\beta t E Y_{1}\right. \\
\quad+y) d J(y) \\
\leq \int_{y-\beta t E Y_{1}<-\epsilon(t) \beta t E Y_{1}} P\left(S_{N(t)}-\mu \lambda(t)>x\right. \\
\left.\quad-\beta t E Y_{1}\right) d J(y) \\
\leq \int_{y-\beta t E Y_{1}<-\epsilon(t) \beta t E Y_{1}} P\left(S_{N(t)}-\mu \lambda(t)\right. \\
\leq L_{F}^{-2} \lambda(t) \bar{F}\left(x\left(1-\frac{E Y_{1}}{\gamma}\right)\right) \\
\quad \times P\left(\sum_{i=1}^{M(t)} Y_{i}-\beta t E Y_{1}<-\epsilon(t) \beta t E Y_{1}\right) \\
\leq C \lambda(t) \bar{F}(x) o(1) \\
=o(\lambda(t) \bar{F}(x)),
\end{aligned}
$$

where $C>0$ is a constant, the last second step holds by $F \in$ $\mathscr{L} \cap \mathscr{D}$ and relation (67).

Lemma 19. For $\epsilon(t)$ in (67), one has for $\gamma>E Y_{1}$ that

$$
\begin{aligned}
L_{F}^{3} \lambda(t) \bar{F}(x) \leqslant I_{2}=\int_{\left|y-\beta t E Y_{1}\right| \leq \epsilon(t) \beta t E Y_{1}} P\left(S_{N(t)}-\mu \lambda(t)>x\right. \\
\left.-\beta t E Y_{1}+y\right) d J(y) \\
\leqslant L_{F}^{-3} \lambda(t) \bar{F}(x)
\end{aligned}
$$

holds uniformly for $x \geq \gamma \lambda(t) \vee \gamma \beta t$.

Proof. For any $x \geq \gamma \lambda(t) \vee \gamma \beta t$ and $\left|y-\beta t E Y_{1}\right| \leq \epsilon(t) \beta t E Y_{1}$, noting that $\epsilon(t) \rightarrow 0$ as $t \rightarrow \infty$, we have for sufficient large $t$,

$$
\begin{aligned}
x-\beta t E Y_{1}+y & \geq x-\epsilon(t) \beta t E Y_{1} \geq\left(1-\frac{\epsilon(t) E Y_{1}}{\gamma}\right) x \\
& >\left(1-\frac{E Y_{1}}{\gamma}\right) x .
\end{aligned}
$$

Again using Theorem 9, we conclude that

$$
L_{F}^{2} \lesssim \frac{P\left(S_{N(t)}-\mu \lambda(t)>x-\beta t E Y_{1}+y\right)}{\lambda(t) \bar{F}\left(x-\beta t E Y_{1}+y\right)} \lesssim L_{F}^{-2}
$$

holds uniformly for $x \geq \gamma \lambda(t)$. Moreover, $\left|y-\beta t E Y_{1}\right| \leq$ $\epsilon(t) \beta t E Y_{1}$, and then $\left|y-\beta t E Y_{1}\right|=o(\lambda(t))$ as $t \rightarrow \infty$; by Lemma 3, we have that

$$
L_{F} \bar{F}(x) \lesssim \bar{F}\left(x-\beta t E Y_{1}+y\right) \lesssim L_{F}^{-1} \bar{F}(x)
$$

holds uniformly for $x \geq \gamma \lambda(t)$.

Hence, combining (67), (74), and (75), we see that

$$
\begin{aligned}
I_{2}= & \int_{\left|y-\beta t E Y_{1}\right| \leq \epsilon(t) \beta t E Y_{1}} P\left(S_{N(t)}-\mu \lambda(t)>x-\beta t E Y_{1}\right. \\
& \quad+y) d J(y) \\
\leq & L_{F}^{-2} \lambda(t) \bar{F}(x) \quad \frac{\bar{F}\left(x-\beta t E Y_{1}+y\right)}{\bar{F}(x)} d J(y) \\
& \times \int_{\left|y-\beta t E Y_{1}\right| \leq \epsilon(t) \beta t E Y_{1}} \frac{}{\lesssim} L_{F}^{-3} \lambda(t) \bar{F}(x) P\left(\left|\sum_{i=1}^{M(t)} Y_{i}-\beta t E Y_{1}\right| \leq \epsilon(t) \beta t E Y_{1}\right) \\
\sim & L_{F}^{-3} \lambda(t) \bar{F}(x) ;
\end{aligned}
$$

symmetrically,

$$
I_{2} \gtrsim L_{F}^{3} \lambda(t) \bar{F}(x) .
$$

Then the proof of Lemma 19 is finished.

Lemma 20. For $\epsilon(t)$ in (67), one has for $\gamma>E Y_{1}$ that

$$
\begin{array}{r}
I_{3}=\int_{y-\beta t E Y_{1}>\epsilon(t) \beta t E Y_{1}} P\left(S_{N(t)}-\mu \lambda(t)>x-\beta t E Y_{1}\right. \\
+y) d J(y)=o(\lambda(t) \bar{F}(x))
\end{array}
$$

holds uniformly for $x \geq \gamma \lambda(t) \vee \gamma \beta$ t.

Proof. For $x \geq \gamma \lambda(t) \vee \gamma \beta t$, by Theorem 9, we see that

$$
\begin{aligned}
I_{3}=\int_{y-\beta t E Y_{1}>\epsilon(t) \beta t E Y_{1}} P\left(S_{N(t)}-\mu \lambda(t)>x-\beta t E Y_{1}\right. \\
+y) d J(y) \\
\leq \int_{y-\beta t E Y_{1}>\epsilon(t) \beta t E Y_{1}} P\left(S_{N(t)}-\mu \lambda(t)>x\right) d J(y) \\
\leq L_{F}^{-2} \lambda(t) \bar{F}(x) P\left(\sum_{i=1}^{M(t)} Y_{i}-\beta t E Y_{1}>\epsilon(t) \beta t E Y_{1}\right) \\
=o(\lambda(t) \bar{F}(x)) ;
\end{aligned}
$$

the last equality is from (67).

\section{Acknowledgments}

The first author is supported by National Science Foundation of China (nos. 10801124 and 11171321) and the Fundamental Research Funds for the Central Universities (no. WK 2040170006). 


\section{References}

[1] D. B. H. Cline and T. Hsing, Large Deviation Probabilities for Sums and Maxima of Random Variables with Heavy or Subexponential Tails, Texas A\&M University, 1991.

[2] C. Klüppelberg and T. Mikosch, "Large deviations of heavytailed random sums with applications in insurance and finance," Journal of Applied Probability, vol. 34, no. 2, pp. 293-308, 1997.

[3] Q. H. Tang, C. Su, T. Jiang, and J. S. Zhang, "Large deviations for heavy-tailed random sums in compound renewal model," Statistics \& Probability Letters, vol. 52, no. 1, pp. 91-100, 2001.

[4] K. W. Ng, Q. Tang, J. A. Yan, and H. Yang, "Precise large deviations for sums of random variables with consistently varying tails," Journal of Applied Probability, vol. 41, no. 1, pp. 93-107, 2004.

[5] D. G. Konstantinides and F. Loukissas, "Precise large deviations for consistently varying-tailed distributions in the compound renewal risk model," Lithuanian Mathematical Journal, vol. 50, no. 4, pp. 391-400, 2010.

[6] F. Loukissas, "Precise large deviations for long-tailed distributions," Journal of Theoretical Probability, vol. 25, no. 4, pp. 913924, 2012.

[7] Y. Chen and W. Zhang, "Large deviations for random sums of negatively dependent random variables with consistently varying tails," Statistics \& Probability Letters, vol. 77, no. 5, pp. 530-538, 2007.

[8] Q. Tang, "Insensitivity to negative dependence of the asymptotic behavior of precise large deviations," Electronic Journal of Probability, vol. 11, no. 4, pp. 107-120, 2006.

[9] Y. Chen, K. C. Yuen, and K. W. Ng, "Precise large deviations of random sums in presence of negative dependence and consistent variation," Methodology and Computing in Applied Probability, vol. 13, no. 4, pp. 821-833, 2011.

[10] K. Wang, Y. Yang, and J. Lin, "Precise large deviations for widely orthant dependent random variables with dominatedly varying tails," Frontiers of Mathematics in China, vol. 7, no. 5, pp. 919932, 2012.

[11] L. Liu, "Precise large deviations for dependent random variables with heavy tails," Statistics \& Probability Letters, vol. 79, no. 9, pp. 1290-1298, 2009.

[12] P. Embrechts, C. Klüppelberg, and T. Mikosch, Modelling Extremal Events for Insurance and Finance, vol. 33 of Applications of Mathematics, Springer, Berlin, Germany, 1997.

[13] T. Rolski, H. Schmidli, V. Schmidt, and J. Teugels, Stochastic Processes for Insurance and Finance, Wiley Series in Probability and Statistics, John Wiley \& Sons, New York, NY, USA, 1999.

[14] Q. Tang and G. Tsitsiashvili, "Precise estimates for the ruin probability in finite horizon in a discrete-time model with heavy-tailed insurance and financial risks," Stochastic Processes and Their Applications, vol. 108, no. 2, pp. 299-325, 2003.

[15] N. H. Bingham, C. M. Goldie, and J. L. Teugels, Regular Variation, vol. 27 of Encyclopedia of Mathematics and Its Applications, Cambridge University Press, Cambridge, UK, 1987.

[16] Y. Hu, "Large deviations for generalized compound poisson risk models and its bankruptcy moments," Science in China A, vol. 47, no. 2, pp. 311-319, 2004.

[17] P. M. Matuła, "A note on the almost sure convergence of sums of negatively dependent random variables," Statistics \& Probability Letters, vol. 15, no. 3, pp. 209-213, 1992. 


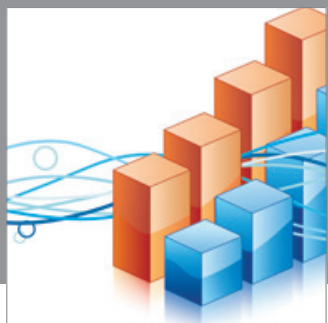

Advances in

Operations Research

mansans

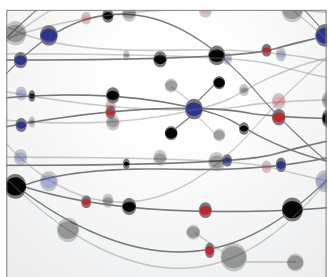

The Scientific World Journal
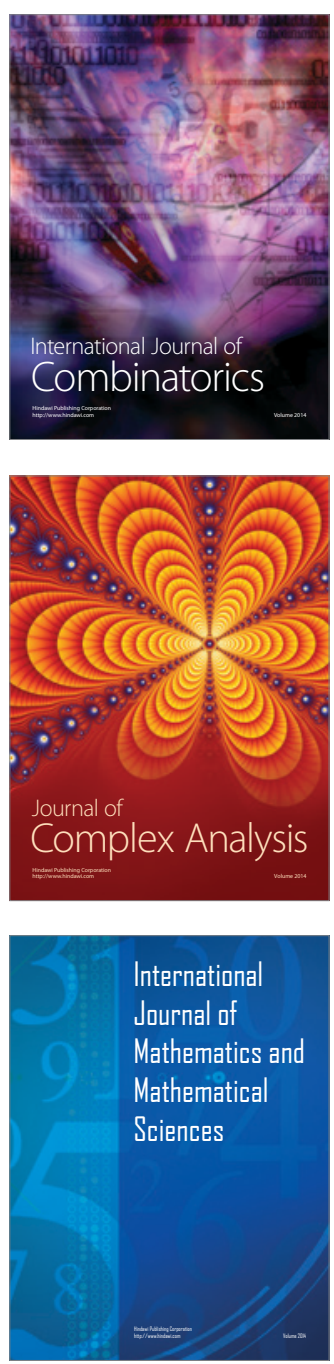
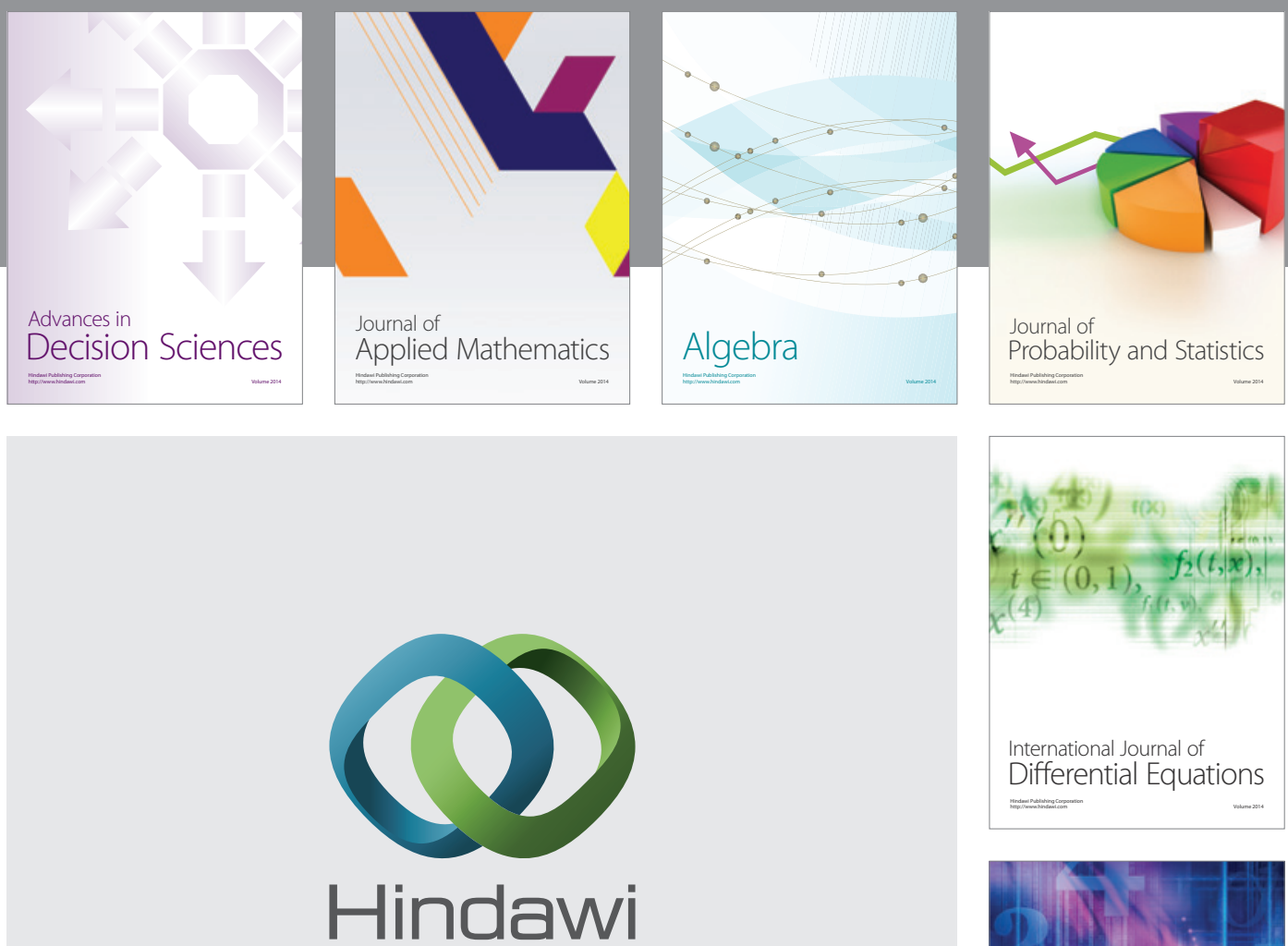

Submit your manuscripts at http://www.hindawi.com
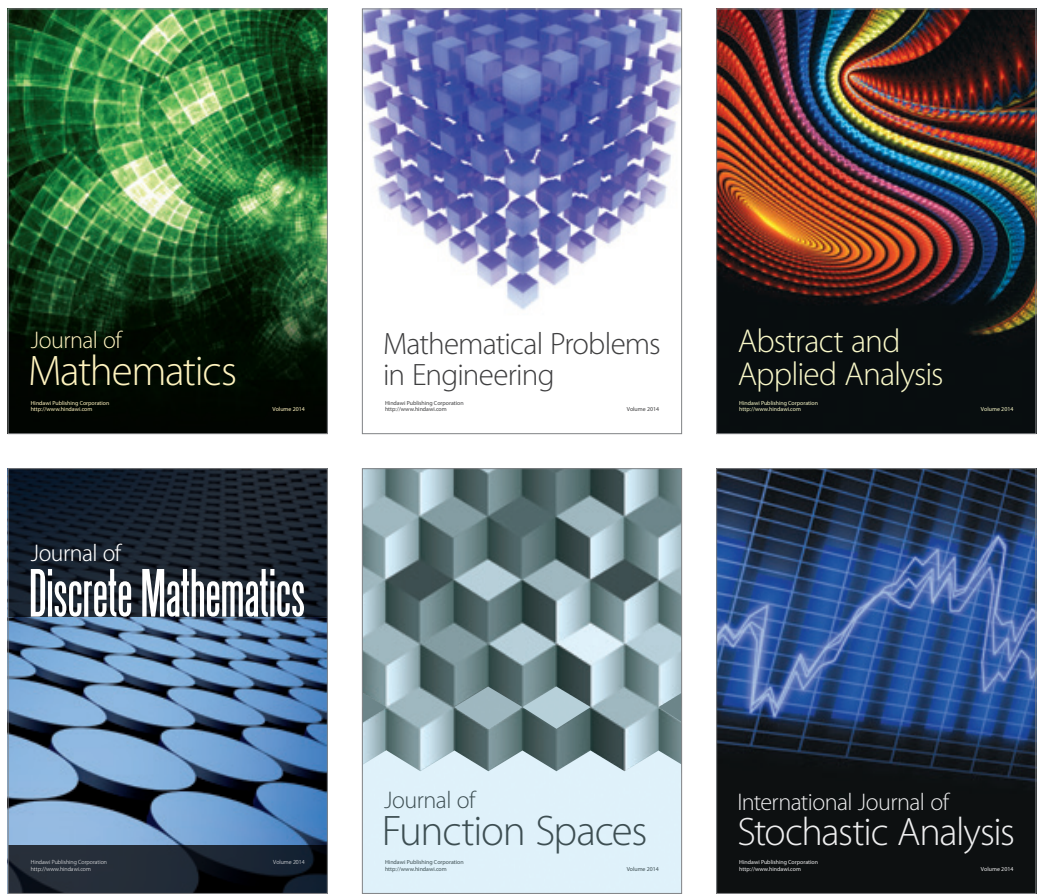

Journal of

Function Spaces

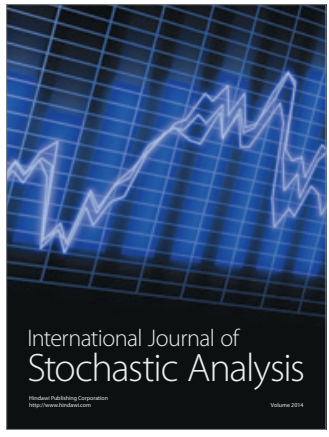

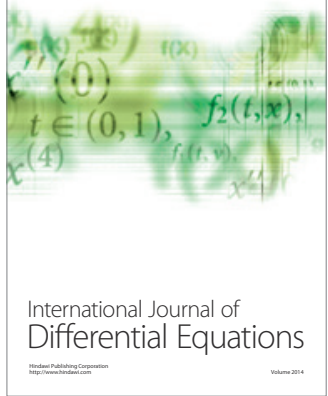
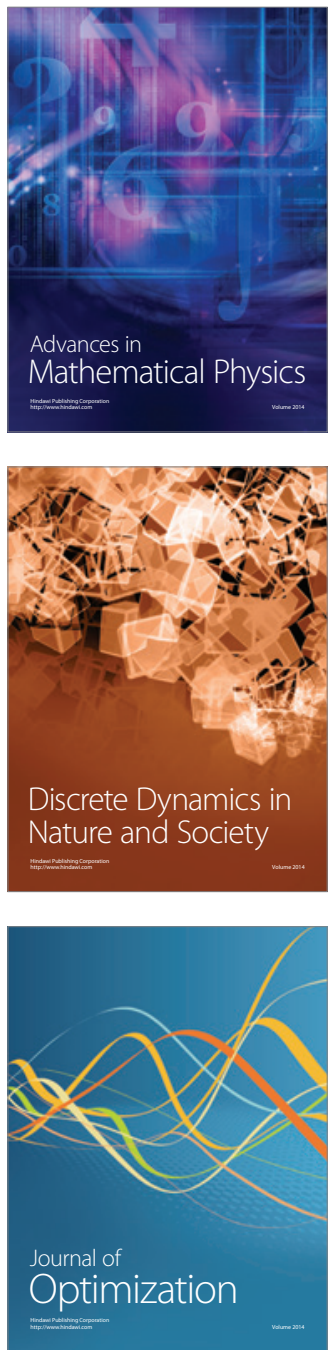